\title{
The Association between the Use of Dietary Supplement and Psychological Status of Cancer Survivors in Korea: A Cross-Sectional Study
}

\author{
Han Rim Lee', Yun-Mi Song ${ }^{1, *}$, Keun Hye Jeon ${ }^{2}$, In Young $\mathrm{Cho}^{3}$ \\ 'Department of Family Medicine, Samsung Medical Center, Sungkyunkwan University School of Medicine, Seoul, Korea \\ ${ }^{2}$ Department of Family Medicine, CHA Gumi Medical Center, CHA University, Gumi, Korea \\ ${ }^{3}$ Department of Family Medicine, Kangbuk Samsung Hospital, Sungkyunkwan University School of Medicine, Seoul, Korea
}

\begin{abstract}
Background: Dietary supplements (DS) use is known to be common among cancer survivors. However, detailed information on the factors influencing DS use seems insufficient, including cancer-related and psychological factors.

Methods: Study subjects were 1,852 Korean adult cancer survivors recruited from cancer survivor clinic of two university-affiliated hospitals. Data were collected retrospectively through review of medical records and self-administered questionnaires. Psychological factors were assessed using Hospital Anxiety and Depression Scale (HADS) and Fear of Cancer Recurrence Inventory-Short Form. Factors associated the DS use were evaluated by multiple logistic regression analysis after adjusting for covariates.

Results: The prevalence of long-term DS use was $15.7 \%$ in overall ( $17.5 \%$ in female and $11.6 \%$ in male). Female survivors were 3.14 times (95\% confidence interval [CI], 1.89-5.22) more likely to use DS than male. In male cancer survivors, ever-smoking and previous radiotherapy were positively associated with DS use. In females, breast cancer survivors were 0.32 times less likely to use DS compared with stomach cancer survivors, and survivors with family history of cancer were 1.39 times more likely to use DS than those without. After adjusting for sociodemographic, clinical, and lifestyle factors, survivors with anxiety (HADS $\geq 8$ ) used DS 1.38 times (95\% CI, 1.01-1.91) more frequently, compared with those without anxiety.

Conclusion: Diverse factors such as female sex, cancer treatment modality, smoking history, family history and anxiety status were associated with DS use in Korean cancer survivors. Targeted strategies with consideration of these factors are needed for counseling DS use for cancer survivors.
\end{abstract}

Keywords: Dietary Supplements; Health Behavior; Cancer Survivors; Anxiety; Depression

Received: August 12, 2020, Revised: January 1, 2021, Accepted: February 18, 2021

${ }^{*}$ Corresponding Author: Yun-Mi Song https://orcid.org/0000-0001-9232-5563

Tel: +82-2-3410-2442, Fax: +82-2-3410-0338, E-mail: yunmisong@skku.edu 


\section{INTRODUCTION}

In spite of rapid growing cancer incidence, ${ }^{1,2)}$ mortality from cancer has been gradually decreasing worldwide probably because of the increasing survival of cancer patients caused by improved cancer detection and treatment. ${ }^{3)}$ Therefore, cancer paradigm has been transforming into a chronic illness to well adapt.

After acute stage of cancer care, cancer survivors tend to initiate behavioral change in an attempt to prevent disease and improve health. ${ }^{4)}$ One of the most common behavioral change of cancer survivors is the use of complementary/alternative medicine (CAM) including dietary supplements (DSs). ${ }^{5}$ A DS is a product containing following ingredients: vitamins, minerals, herbs, or other substances, which is taken orally. ${ }^{6,7)}$ Several studies have shown that cancer survivors are more likely to use DS. In a study including American cancer survivors, prevalence of DS use among cancer survivors was higher $(70.4 \%$ versus $51.2 \%$ ) than the prevalence among cancer-free individual. ${ }^{8)}$ DS use is also common among Korean cancer patients. A nation-wide crosssectional Korean study also found that Korean cancer survivors use DS (33.3\%) more frequently than cancer-free individuals (22.1\%). $\left.{ }^{9}\right)$

In previous study on general population, factors associated with more frequent use of DS were female gender $(\mathrm{P}=0.041)$, Caucasian $(\mathrm{P}<0.001)$, and older age (mean age, 56.98 years versus 53.34 years; $\mathrm{P}<0.001)$, and higher number of chronic conditions $(\mathrm{P}=0.004) .{ }^{10)}$ In a study of American cancer survivors, female gender (odds ratio [OR], 1.72; 95\% confidence interval [CI], 1.25-2.36) and higher education levels (OR, 5.44; 95\% CI, 2.98-9.93) were associated with DS use. ${ }^{11)}$ For Korean female cancer survivors, educational achievement higher than elementary school level, moderate physical activity, low vegetable intake, and high circulating vitamin D levels were associated with supplement use. ${ }^{9)}$ For Korean male cancer survivors, living in an urban area, no consumption of alcohol, and lower energy intake were associated with DS use. ${ }^{9)}$ However, cancer related characteristics related with DS use such as cancer site and cancer stage could not be evaluated because of the small number of cancer survivors (260 women and 140 men) included in the Korean study. In addition, to our knowledge, no study has evaluated the relation between psychological factors and DS use in Korean cancer survivors.

Fear of cancer recurrence (FCR) is a possible mechanism for behavioral change such as DS use among cancer survivors. Compared with the cancer survivors without FCR, cancer survivors with low (OR, 1.97; 95\% CI, 1.36-2.85) or high (OR, 2.40; 95\% CI, 1.33-4.32) FCR were more likely to have healthier habits in the United States. ${ }^{12)}$

Cancer survivors commonly have psychological distress such as depression and anxiety. ${ }^{13)}$ A study in India reported that $33.5 \%$ of cancer patients had diagnosed with either anxiety or depressive disorder. ${ }^{14)}$ The relation between psychological status and the use of CAM has been scarcely studied. ${ }^{15,16)}$ In a study in Turkey showed no correlation between usage of CAM and anxiety and depression among breast cancer survivors. ${ }^{15)}$ In an Italian study, the levels of anxiety and depression did not differ according to the CAM use. ${ }^{16)}$ However, the number of cancer patients who reported CAM use might be too small (61 among 122,52 among 288) to adequately evaluate the association of CAM use with the psychological status. Moreover, the relation between psychological status and the use of CAM has been scarcely studied in Asian country.

In these regards, we conducted this study to evaluate the associations of the use of DS with a range of factors with special attention to psychological variables, in a large sample of Korean cancer survivors.

\section{METHODS}

\section{Study Participants}

A total of 2,038 adult cancer survivors were recruited for this study, who had visited two university affiliated hospitals for routine surveillance from September 2014 to February 2017. We excluded those who did not provide information related to cancer diagnosis ( $\mathrm{n}=163$ ), cancer treatment ( $n=19)$, and those with missing data for variables measuring psychologic factors such as anxiety, depression, and FCR $(n=4)$. As a result, a total of 1,852 adults aged $\geq 19$ years were included in the analysis. All participants provided an informed consent form. The Institutional Review Board (IRB) of Samsung Medical Center (SMC 2013-07-133) and Seoul National University Hospital (H-1407-066595) approved the study protocol.

\section{Study Variables}

We collected information on DS use, demographic and socioeconomic characteristics, comorbidities, health behavioral factors and psychological status of study participants using a self-administered questionnaire. A trained research assistant supplemented incompletely answered questions through an interview.

We collected information on the type, frequency and duration of usage of DS by providing a predetermined list of DS (multivitamin, vitamin C, vitamin D, calcium, iron, omega-3, baby aspirin, etc.) which was commonly used among Koreans. If a participant was using other DS than those listed, he/she could report them additionally. We defined long-term DS use as the use of any DS at least once a week for 6 months or longer. Aspirin use for secondary prevention of ischemic heart disease and ischemic stroke and iron supplementation for treating iron deficiency anemia were not counted as DS use.

We categorized monthly income into three levels ( $\geq 4,000,000$ won, $2,000,000-3,999,000$ won, or $<2,000,000$ won). Education was categorized into three levels ( $\geq$ graduate degree, high school graduate, or $\leq$ middle school graduate). Marital status had two groups (living with spouse/or partner, or not). Smoking status was grouped into never smoker or ever smoker. We calculated the amount of pure alcohol consumption per week on the basis of the amount of liquor consumption at one episode and the frequency of drinking per week. We then categorized alcohol consumption into two levels ( $<7 \mathrm{~g} / \mathrm{wk}, \geq 7 \mathrm{~g} / \mathrm{wk}$ ). We defined regular physical exercise as moderate or higher strength of physical activity for more than 30 minutes each time, with a frequency of twice or more per a week. Comorbidity included diabetes, cardio- 
vascular disease, cerebrovascular disease, chronic respiratory disease, chronic liver disease, and chronic renal disease. We then categorized comorbidity into three groups on the basis of the number of coexisting comorbidity $(0,1$, or $\geq 2)$. Information on family history of cancer was categorized into two groups (yes, no).

We collected cancer-related information, which includes cancer site, cancer stage, time since cancer diagnosis, and cancer treatment. Cancer stage was categorized into three groups (stage 0 [defined as carcinoma in situ] or stage 1, stage 2, stage 3, or stage 4). Hospital Anxiety and Depression Scale (HADS) was used to assess psychological status of participants as HADS is a widely using psychometrics for screening anxiety and depression in patients. ${ }^{17)}$ We assessed cut off value both $\geq 8$ on HADS-anxiety and HADS-depression for detecting generalized anxiety disorders and major depressive episodes. ${ }^{18)} \mathrm{We}$ assessed the presence of FCR using cut off value $\geq 13$ of severity subscale of the Fear of Cancer Recurrence Inventory (FCRI). ${ }^{19,20)}$ The FCRI is a multidimensional questionnaire composed of 42 items with seven subscale components of FCR, and the FCR severity subscale is used as the FCR Short Form. ${ }^{19)}$

\section{Statistical Analysis}

We looked at distribution of long-term use of commonly used DS by sex. We compared demographic and socioeconomic characteristics, health behavioral factors, and clinical characteristics according to the long-term use of DS by chi-square test or Fisher's exact test.

We estimate ORs and 95\% CIs to evaluate factors associated with the use of DS in cancer survivors, by multivariable logistic regression analysis after adjusting for age, sex, education, marital status, smoking, alcohol intake, physical activity, comorbidity, family cancer history, cancer type, cancer stage, cancer treatment modality, and time since cancer diagnosis. As we found a significant difference in the use of DS by sex, we did all analysis separately in male survivors and female survivors.

Finally, to evaluate a specific association between each psychologi- cal factor (anxiety, depression, and FCR) and DS use, we conducted additional analysis which further included each psychological factor in the multiple logistic regression analysis. To evaluate modifying effect of covariates on the association between psychological factors and DS use, we repeatedly conducted the analysis separately by sex, by cancer site (breast and stomach), and by time since cancer diagnosis. The level of statistical significance was a P-value $<0.05$ in two-sided tests. We used SAS ver. 9.4 (SAS Institute Inc., Cary, NC, USA) for all statistical analyses.

\section{RESULTS}

We found that $15.7 \%(n=290)$ of cancer survivors reported long-term use of DS. Female survivors (17.5\%) used DS more commonly than male survivors (11.6\%) (OR, 3.14; 95\% CI, 1.89-5.22). Most commonly used DS was multi-vitamin ( $6.4 \%$ in male, $7.1 \%$ in female), which was followed by vitamin C (3.3\% in male, $5.8 \%$ in female), calcium supplement ( $1.2 \%$ in male, $5.9 \%$ in female), omega-3 (2.6\% in male, $4.2 \%$ in female), vitamin D (1.0\% in male, $4.4 \%$ in female), and iron $(2.1 \%$ in male, $1.9 \%$ in female) (Figure 1).

In Table 1, demographic and clinical characteristics were compared according to the long-term use of DS. Female survivors tended to less frequently use DS with increasing age. Female survivors with family history of cancer were more likely to use DS. In male survivors, eversmoking was related with use of DS.

In Table 2, cancer related characteristics were compared according to the long-term use of DS. In overall, with increasing stage of cancer, prevalence of DS use increased. In male survivors, DS use did not differ by cancer type. However, in female DS use differed by cancer type: most prevalent among thyroid cancer survivors (29.8\%), followed by lung $(22.0 \%)$, stomach $(21.6 \%)$, and colorectal cancer survivors (15.1\%). Male survivors' chemotherapy or radiotherapy were related with more frequent use of DS. Female survivors who received hormone therapy used DS less frequently than those who did not.

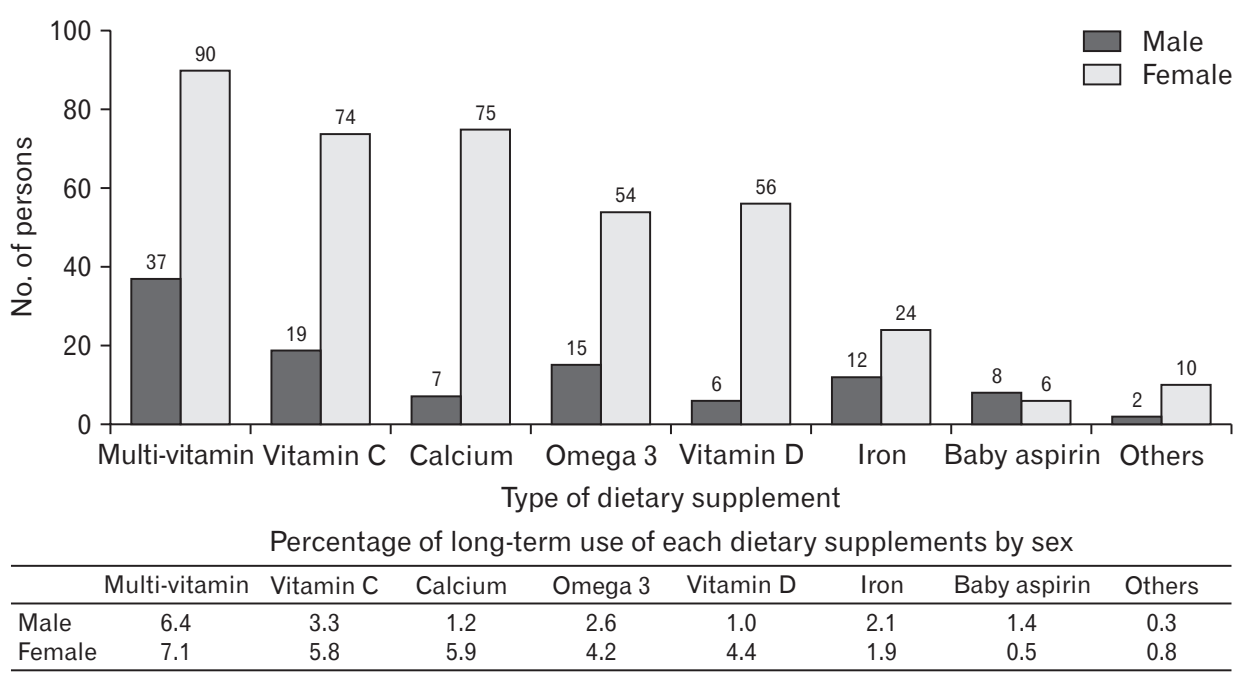

Others contain coenzyme Q10, lactobacillus, propolis, and ginseng.
Figure 1. Distribution of long-term ( $\geq 6$ months) use of dietary supplements by sex among 1,852 Korean adulthood cancer survivors. 


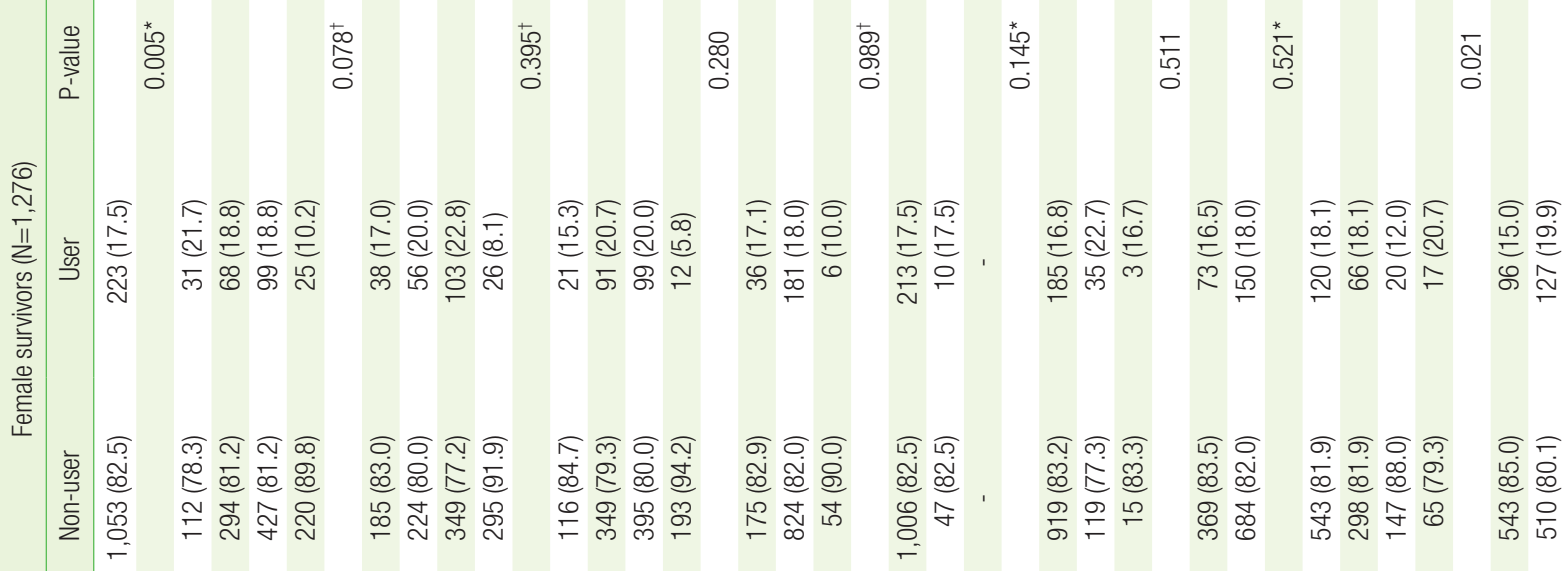

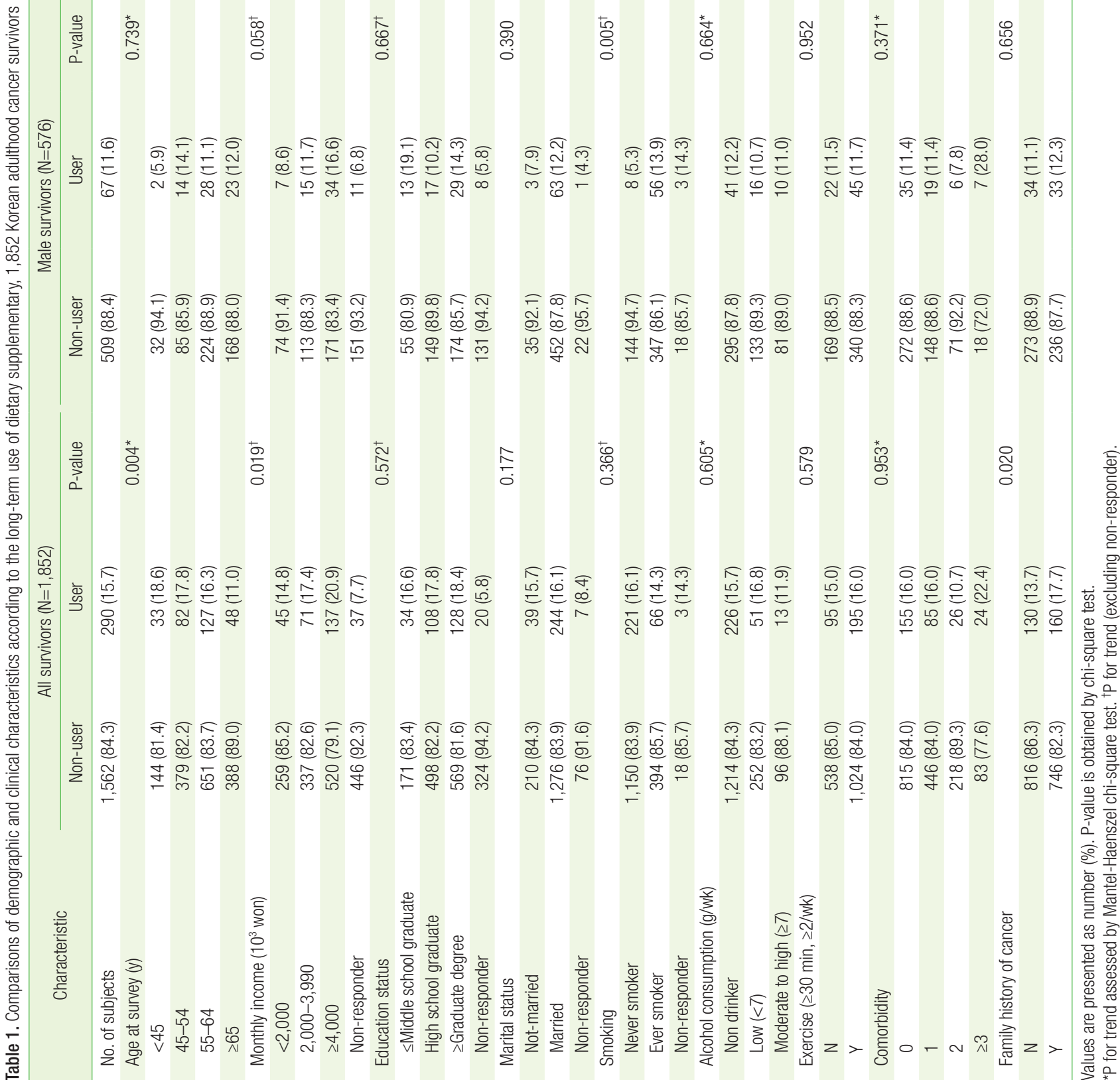




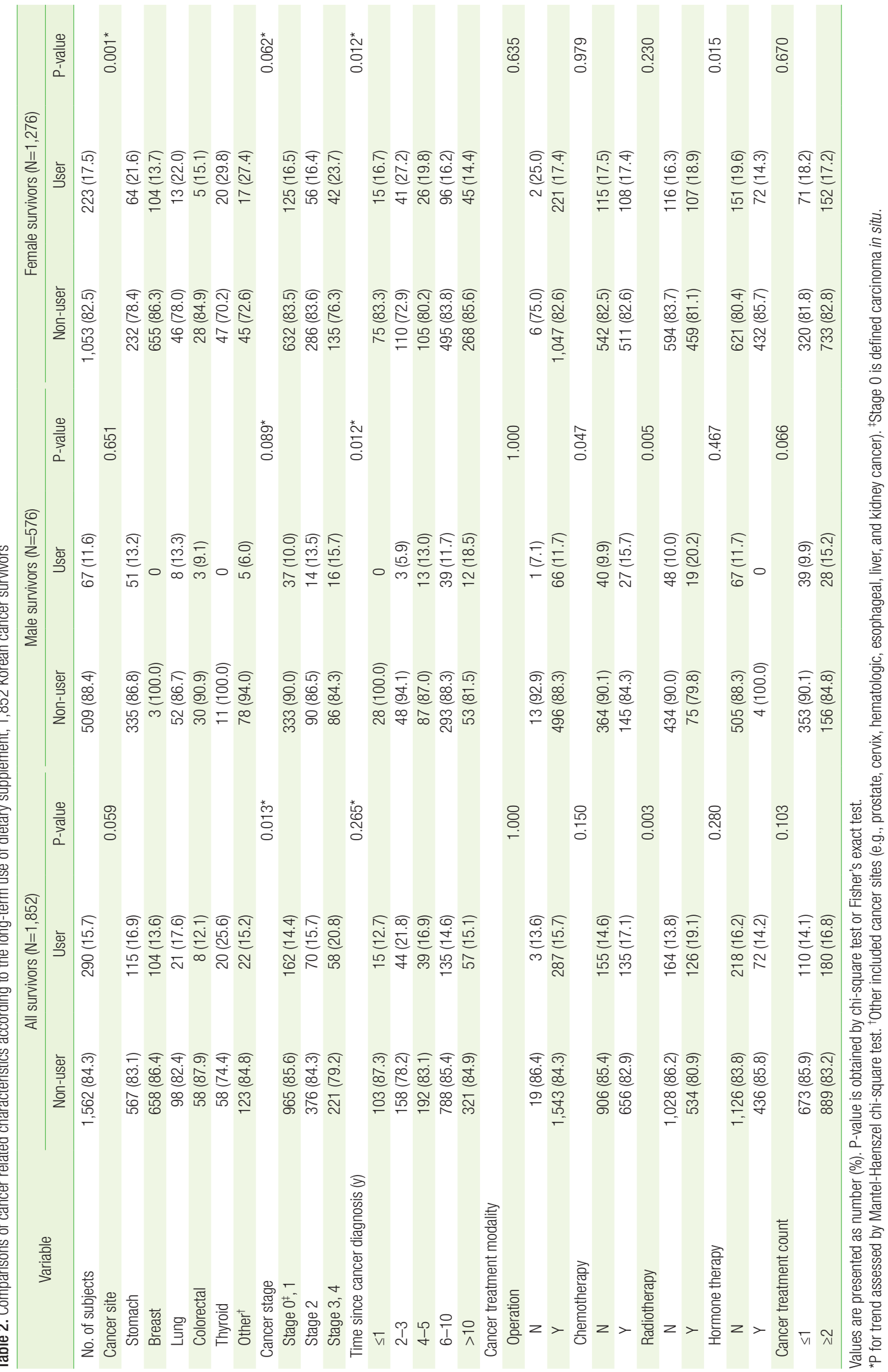


Table 3. Multivariable adjusted associations of long-term use of dietary supplement with demographic, clinical and cancer related characteristics, 1,852 Korean cancer survivors

\begin{tabular}{|c|c|c|c|}
\hline Variable & All survivors & Male cancer survivors & Female cancer survivors \\
\hline \multicolumn{4}{|l|}{ Sex } \\
\hline Male & 1 & & \\
\hline Female & $3.14(1.89-5.22)$ & & \\
\hline \multicolumn{4}{|l|}{ Age at survey (y) } \\
\hline$<45$ & 1 & 1 & 1 \\
\hline $45-54$ & $1.13(0.70-1.81)$ & $2.92(0.57-14.82)$ & $1.02(0.61-1.69)$ \\
\hline $55-64$ & $1.22(0.76-1.95)$ & $2.34(0.48-11.38)$ & $1.15(0.69-1.93)$ \\
\hline$\geq 65$ & $1.01(0.58-1.75)$ & $3.72(0.73-18.88)$ & $0.68(0.35-1.31)$ \\
\hline \multicolumn{4}{|l|}{ Education status } \\
\hline$\leq$ Middle school graduate & 1 & 1 & 1 \\
\hline High school graduate & $1.05(0.68-1.64)$ & $0.54(0.23-1.24)$ & $1.39(0.81-2.39)$ \\
\hline$\geq$ Graduate degree & $1.22(0.77-1.89)$ & $0.88(0.40-1.93)$ & $1.44(0.82-2.52)$ \\
\hline \multicolumn{4}{|l|}{ Marital status } \\
\hline Not-married & 1 & 1 & 1 \\
\hline Married & $0.99(0.90-1.08)$ & $0.91(0.71-1.16)$ & $1.00(0.90-1.12)$ \\
\hline \multicolumn{4}{|l|}{ Smoking } \\
\hline Never smoker & 1 & 1 & 1 \\
\hline Ever smoker & $1.50(0.93-2.42)$ & $2.61(1.15-5.93)$ & $0.91(0.43-1.94)$ \\
\hline \multicolumn{4}{|l|}{ Alcohol consumption (g/wk) } \\
\hline Non drinker & 1 & 1 & 1 \\
\hline Low $(<7)$ & $1.11(0.77-1.58)$ & $0.74(0.38-1.45)$ & $1.28(0.83-1.98)$ \\
\hline Moderate to high ( $\geq 7)$ & $0.89(0.46-1.72)$ & $0.76(0.33-1.73)$ & $0.78(0.21-2.94)$ \\
\hline \multicolumn{4}{|l|}{ Exercise ( $\geq 30 \mathrm{~min}, \geq 2 / w \mathrm{k}$ ) } \\
\hline $\mathrm{N}$ & 1 & 1 & 1 \\
\hline Y & $1.22(0.92-1.63)$ & $1.15(0.63-2.13)$ & $1.27(0.91-1.76)$ \\
\hline \multicolumn{4}{|l|}{ Comorbidity } \\
\hline 0 & 1 & 1 & 1 \\
\hline 1 & $1.03(0.76-1.39)$ & $0.94(0.49-1.78)$ & $1.04(0.73-1.48)$ \\
\hline$\geq 2$ & $1.00(0.69-1.45)$ & $1.29(0.61-2.72)$ & $0.99(0.64-1.54)$ \\
\hline \multicolumn{4}{|l|}{ Family history of cancer } \\
\hline $\mathrm{N}$ & 1 & 1 & 1 \\
\hline Y & $1.34(1.03-1.74)$ & $1.14(0.65-2.00)$ & $1.39(1.03-1.89)$ \\
\hline \multicolumn{4}{|l|}{ Cancer site } \\
\hline Stomach & 1 & 1 & 1 \\
\hline Breast & $0.29(0.18-0.47)$ & - & $0.32(0.19-0.53)$ \\
\hline Lung & $0.91(0.53-1.54)$ & $0.73(0.31-1.71)$ & $0.98(0.49-2.00)$ \\
\hline Colorectal & $0.46(0.21-1.04)$ & $0.40(0.11-1.47)$ & $0.43(0.15-1.27)$ \\
\hline Thyroid & $1.03(0.56-1.90)$ & $<0.01(<0.01->999.99)$ & $1.29(0.67-2.49)$ \\
\hline Other* & $0.62(0.36-1.07)$ & $0.25(0.08-0.75)$ & $0.92(0.46-1.82)$ \\
\hline \multicolumn{4}{|l|}{ Cancer stage } \\
\hline Stage $0^{\dagger}, 1$ & 1 & 1 & 1 \\
\hline Stage 2 & $1.08(0.74-1.57)$ & $1.75(0.66-4.60)$ & $1.02(0.67-1.55)$ \\
\hline Stage 3,4 & $1.32(0.87-2.02)$ & $1.81(0.64-5.13)$ & $1.28(0.79-2.08)$ \\
\hline \multicolumn{4}{|c|}{ Time since cancer diagnosis $(y)$} \\
\hline$\leq 1$ & 1 & 1 & 1 \\
\hline $2-3$ & $1.26(0.26-6.06)$ & $<0.01(<0.01->999.99)$ & $1.60(0.31-8.24)$ \\
\hline $4-5$ & $3.65(0.60-22.18)$ & $8.23(0.51-133.01)$ & $2.23(0.20-25.28)$ \\
\hline $6-10$ & $0.76(0.09-6.65)$ & $1.06(0.10-11.79)$ & $<0.01(<0.01->999.99)$ \\
\hline$>10$ & $0.74(0.25-2.19)$ & $0.66(0.08-5.41)$ & $0.83(0.23-3.01)$ \\
\hline \multicolumn{4}{|l|}{ Cancer treatment modality } \\
\hline \multicolumn{4}{|l|}{ Surgery } \\
\hline $\mathrm{N}$ & 1 & 1 & 1 \\
\hline Y & $1.18(0.32-4.40)$ & $2.33(0.19-28.88)$ & $0.49(0.08-3.01)$ \\
\hline \multicolumn{4}{|l|}{ Chemotherapy } \\
\hline $\mathrm{N}$ & 1 & 1 & 1 \\
\hline Y & $0.94(0.60-1.47)$ & $2.06(0.31-13.66)$ & $0.93(0.58-1.49)$ \\
\hline
\end{tabular}


Table 3. Continued

\begin{tabular}{lccc}
\hline \multicolumn{1}{c}{ Variable } & All survivors & Male cancer survivors & Female cancer survivors \\
\hline Radiotherapy & & 1 & 1 \\
N & 1 & $3.56(1.37-9.25)$ & $1.40(0.92-2.11)$ \\
Y & $1.67(1.15-2.43)$ & 1 & 1 \\
Hormone therapy & 1 & $<0.01(<0.01->999.99)$ & $0.92(0.59-1.44)$ \\
N & $0.93(0.60-1.44)$ & 1 & 1 \\
Y & 1 & $0.29(0.03-2.44)$ & $1.63(0.87-3.06)$ \\
Cancer treatment count & $1.35(0.77-2.35)$ & & \\
$\leq 1$ & & & \\
$\geq 2$ & & & \\
\end{tabular}

Values are presented as OR $(95 \% \mathrm{Cl})$. ORs $(95 \% \mathrm{Cl})$ were estimated by multiple logistic regression analysis after adjusting for age group, sex (if applicable), education, marital status, smoking, alcohol intake, physical activity, comorbidity, family cancer history, cancer type, cancer stage, time since cancer diagnosis, and cancer treatment. $\mathrm{OR}$, odds ratio; $\mathrm{Cl}$, confidence interval; FCR, fear of cancer recurrence.

${ }^{*}$ Other included cancer sites (e.g., prostate, cervix, hematologic, esophageal, liver, and kidney cancer). ${ }^{\dagger}$ Stage 0 is defined carcinoma in situ.

Table 3 shows the independent associations of long-term use of DS with demographic, clinical and cancer related characteristics. Female cancer survivors were 3.14 times more likely to use DS compared with male survivors. In female, compared with stomach cancer survivor, breast cancer survivors less used DS (OR, 0.32; 95\% CI, 0.19-0.53) and those who had family history of cancer were related with more frequent use of DS (OR, 1.39; 95\% CI, 1.03-1.89). In male, survivors who had ever smoked were more likely to use DS than never smoker (OR, 2.61; 95\% CI, 1.15-5.93) and who had undergone radiotherapy for cancer treatment were more likely to use DS (OR, 3.56; 95\% CI, 1.37-9.25).

Table 4 shows associations of long-term DS use with status of anxiety, depression, and FCR severity. In all participants, survivors with anxiety were 1.38 times (95\% CI, 1.01-1.91) more likely to use DS, compared to the survivors without anxiety. There was no significant difference in the association between the psychological factors and DS use, by different sex, by different cancer sites (breast and stomach), and by different time since cancer diagnosis ( $\mathrm{P}$ for interaction $>0.05$ ).

\section{DISCUSSION}

In this Korean cancer survivor study, we found that cancer type and cancer treatment modality among cancer-related characteristics were associated with DS use. Among lifestyle factors, smoking habit in male survivors and family history of cancer in female survivor were significantly associated. Among psychological factors, anxiety, but not depression or FCR, was associated with DS use.

Previous studies have reported that the use of DS or CAM after cancer diagnosis is very common. In American study, overall prevalence rate of DS use was $75 \%$ among cancer survivors. ${ }^{8)}$ Other French cohort study reported $62 \%$ of female and $29 \%$ of male cancer patients use DS. ${ }^{21)}$ In Korean study, 33.3\% of cancer survivor reported to use DS. ${ }^{9)}$ In our study, we found $15.7 \%$ of cancer survivors were using DS at least for 6 months, which seems to be much lower than the rate reported previous Korean study. We think this difference in the prevalence of DS use between our study and the previous Korean study by Song et al. ${ }^{9)}$ was caused by the different definition of DS use between the stud- ies. We defined examined long-term ( $\geq 6$ months use of DS) while Song et al. ${ }^{9)}$ examined the use of any DS on the preceding day of the survey.

Participants taking DS were more often to be female, young, with higher income level. These findings were consistent with the findings of previous studies. In American study, female gender (OR, 1.72; 95\% CI, 1.25-2.36) and higher-education levels (OR, 5.44; 95\% CI, 2.989.93) were associated with DS use. ${ }^{11)}$ In previous Korean study, Korean female cancer survivors with higher educational achievement than elementary school level, moderate physical activity, low vegetable intake, and high circulating vitamin D levels were more likely to use DS. In the same study male cancer survivors who were never drinker used DS more frequently. ${ }^{9)}$

We found that ever smoking was associated with DS use among male cancer survivors. On the contrary, DS use was more commonly associated with a healthier lifestyle in previous studies. For male cancer survivors, nonuse of alcohol was associated with higher supplement use (OR, 7.88; 95\% CI, 1.45-42.82), compared with ever drinking. ${ }^{9)}$ DS use was significantly lower among former smokers (OR, 0.57; 95\% CI, 0.37-0.87) compare with never smokers in the French cohort study of cancer survivors. ${ }^{21)}$ As a reason for the higher use of DS among ever-smokers than never-smokers, we think desire to overcome health problems associated with smoking by using DS might have caused the higher rate of DS use. This discrepant result could have derived from that we didn't consider the behavioral change like quit smoking at the time of study.

In our study, breast cancer survivors used DS less frequently compared with female stomach cancer survivors (OR, 0.31; 95\% CI, 0.190.50). As for the reason of this finding, we assume that health knowledge commonly shared among stomach and breast cancer survivors might have influenced their decision on DS use. It is well known that post gastrectomy anemia is common, so that stomach cancer survivors might have been more likely to take iron supplementation regardless of anemia diagnosis. This hypothesis could be supported by the finding that among 62 (72.9\%) among 85 iron supplement users were stomach cancer survivors in our study, even though iron use of survivors with iron deficiency anemia was not counted. We also thought 


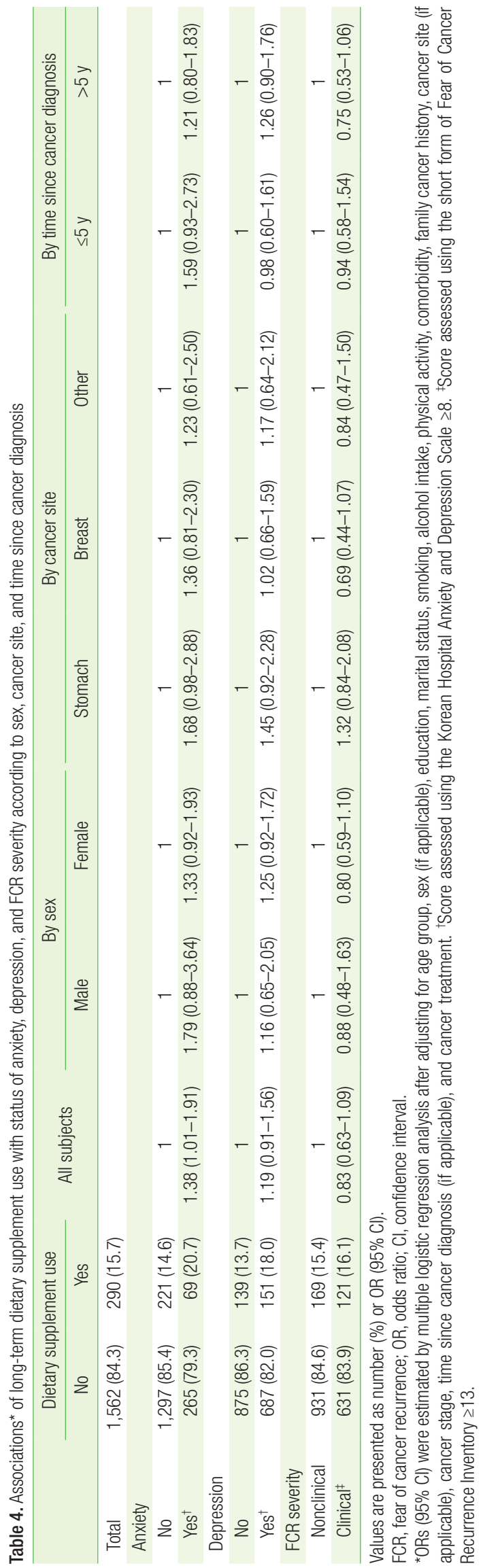

another possibility that known adverse effect of DS use on breast cancer might have reduced the DS use. An American study showed that the use of any antioxidant supplement (vitamins A, C, and E, carotenoids, coenzyme Q10) during chemotherapy for breast cancer was associated with an increased risk of cancer recurrence (hazard ratio [HR], 1.41; 95\% CI, 0.98-2.04; P=0.06). Vitamin B12 use was associated with worse disease-free survival ( $\mathrm{HR}, 1.83$; 95\% CI, 1.15-2.92; $\mathrm{P}<0.01$ ) and overall survival (HR, 2.04; 95\% CI, 1.22-3.40; $\mathrm{P}<0.01$ ). Iron was significantly associated with cancer recurrence (HR, 1.79; 95\% CI, 1.202.67; $\mathrm{P}<0.01){ }^{22)}$ This information might have hindered breast cancer survivors from DS use even after completing acute cancer treatment. However, we could not examine these assumptions because of the lack of qualitative data.

To our knowledge, this study was the first study to have examined the association between DS use and cancer treatment modality. We found that radiotherapy was positively associated with DS use in male cancer survivors. Several reasons could be thought for DS use or CAM use among cancer survivors: to improve immune system, ${ }^{11)}$ to prevent cancer recurrence, to increase treatment efficacy and to reduce treatment-related symptoms among patients under active cancer treatment. ${ }^{23)}$ Considering that time lapse after cancer diagnosis was longer than 1 year for most study participants in our study, other reason than immune system or cancer treatment efficacy could have caused the higher DS use in survivors after radiotherapy. In our study, the majority of male cancer survivors who had received radiation therapy was stomach (45.7\%) and colorectal cancer survivors (14.9\%). Given that delayed radiation-induced gastrointestinal (GI) toxicity is common among cancer survivors who had received radiation therapy for the treatment of intra-abdominal tumors. ${ }^{24)}$ DS use to relieve GI symptoms might have been more prevalent among the cancer survivors of stomach and colorectal cancers after radiation therapy.

To our knowledge, this is the first study to examine the association of psychologic factors (anxiety, depression and FCR) with DS use among Asian cancer survivors. We found that anxiety was associated with 1.38-times higher use of DS. A study in the United States similarly showed that a history of depression or anxiety was associated with higher complementary health approaches (OR, 1.87; 95\% CI, 1.133.09) in multivariable adjusted analysis. ${ }^{25)}$ However, we could not directly compare the finding of the US study with ours, because the US study did not examine anxiety and depression separately. We couldn't find any association between FCR level and DS use, which is incompatible with the findings of a study in the United States that CAM use was associated with FCR (OR, 1.96; 95\% CI, 1.38-2.78). ${ }^{26)}$

The present study has several limitations. First, the study participants were recruited from the patients who visited survivorship clinic of university affiliated hospitals. Therefore, they might not well represent Korean cancer survivors and there could occur selection bias. Second, the data on DS use might not accurately reflect actual usage, because we collected the data using a self-administered questionnaire. Third, we could not compare DS usage between cancer survivors and general population because our study subjects were limited to cancer 
survivors. Fourth, the current study targeted only the long-term use of DS, and the findings of this study might not applicable to short term use of DS by cancer survivors. Fifth, given the cross-sectional study design, we cannot exclude the possibility that the associations observed in the present study are reverse cause-effect relationship. Finally, as we did not collect qualitative data on the DS use, we could not interpret some study findings with evidence.

In conclusion, this study in Korean adult cancer survivors revealed that about 1-sixth of cancer survivors tend to use DS for 6 month or longer. Female survivors used DS more frequently than male survivors. Factors associated with long-term use of DS differed between male and female survivors. Among psychological factors, anxiety was significantly associated with DS use of cancer survivors, even after adjusting for a wide range of demographic, clinical and cancer related characteristics. These findings suggest that health education regarding longterm use of DS for cancer survivors may be needed with consideration of demographic, clinical, and psychological characteristics of individual cancer survivors.

\section{CONFLICT OF INTEREST}

No potential conflict of interest relevant to this article was reported.

\section{ACKNOWLEDGMENTS}

This study was supported by a grant from the National R\&D Program for Cancer Control, Ministry of Health \& Welfare, Republic of Korea (1720270).

\section{ORCID}

Han Rim Lee: https://orcid.org/0000-0003-1223-9193

Yun-Mi Song: https://orcid.org/0000-0001-9232-5563

Keun Hye Jeon: https://orcid.org/0000-0003-2294-0637

In Young Cho: https://orcid.org/0000-0002-6240-9026

\section{REFERENCES}

1. Bray F, Ferlay J, Soerjomataram I, Siegel RL, Torre LA, Jemal A. Global cancer statistics 2018: GLOBOCAN estimates of incidence and mortality worldwide for 36 cancers in 185 countries. CA Cancer J Clin 2018; 68:394-424.

2. Jung KW, Won YJ, Kong HJ, Lee ES. Cancer statistics in Korea: incidence, mortality, survival, and prevalence in 2016. Cancer Res Treat 2019;51:417-30.

3. Siegel RL, Miller KD, Jemal A. Cancer statistics, 2019. CA Cancer J Clin 2019;69:7-34.

4. Demark-Wahnefried W, Aziz NM, Rowland JH, Pinto BM. Riding the crest of the teachable moment: promoting long-term health after the diagnosis of cancer. J Clin Oncol 2005;23:5814-30.

5. Miller PE, Vasey JJ, Short PF, Hartman TJ. Dietary supplement use in adult cancer survivors. Oncol Nurs Forum 2009;36:61-8.
6. National Center for Complementary and Integrative Health. Dietary and herbal supplements [Internet]. Bethesda (MD): National Center for Complementary and Integrative Health; 2018 [cited 2020 May 19]. Available from: https://nccih.nih.gov/health/supplements.

7. U.S. Food and Drug Administration. Dietary supplements: what you need to know [Internet]. Silver Spring (MD): U.S. Food and Drug Administration; 2017 [cited 2020 May 19]. Available from: https://www.fda. gov/Food/DietarySupplements/UsingDietarySupplements/ucm109760. htm.

8. Du M, Luo H, Blumberg JB, Rogers G, Chen F, Ruan M, et al. Dietary supplement use among adult cancer survivors in the United States. J Nutr 2020;150:1499-508.

9. Song S, Youn J, Lee YJ, Kang M, Hyun T, Song Y, et al. Dietary supplement use among cancer survivors and the general population: a nation-wide cross-sectional study. BMC Cancer 2017;17:891.

10. Friedman J, Birstler J, Love G, Kiefer D. Diagnoses associated with dietary supplement use in a national dataset. Complement Ther Med 2019;43:277-82.

11. Ferrucci LM, McCorkle R, Smith T, Stein KD, Cartmel B. Factors related to the use of dietary supplements by cancer survivors. J Altern Complement Med 2009;15:673-80.

12. Reed SC, Bell JF, Miglioretti DL, Nekhlyudov L, Fairman N, Joseph JG. Relationships between fear of cancer recurrence and lifestyle factors among cancer survivors. J Cancer Educ 2020;35:669-77.

13. Harrington CB, Hansen JA, Moskowitz M, Todd BL, Feuerstein M. It's not over when it's over: long-term symptoms in cancer survivors: a systematic review. Int J Psychiatry Med 2010;40:163-81.

14. Gopalan MR, Karunakaran V, Prabhakaran A, Jayakumar KL. Prevalence of psychiatric morbidity among cancer patients: hospital-based, cross-sectional survey. Indian J Psychiatry 2016;58:275-80.

15. Kalender ME, Buyukhatipoglu H, Balakan O, Suner A, Dirier A, Sevinc A, et al. Depression, anxiety and quality of life through the use of complementary and alternative medicine among breast cancer patients in Turkey. J Cancer Res Ther 2014;10:962-6.

16. Saini A, Berruti A, Capogna S, Negro M, Sguazzotti E, Picci RL, et al. Prevalence of complementary/alternative medicines (CAMs) in a cancer population in northern Italy receiving antineoplastic treatments and relationship with quality of life and psychometric features. Qual Life Res 2011;20:683-90.

17. Bjelland I, Dahl AA, Haug TT, Neckelmann D. The validity of the Hospital Anxiety and Depression Scale: an updated literature review. J Psychosom Res 2002;52:69-77.

18. Olsson I, Mykletun A, Dahl AA. The Hospital Anxiety and Depression Rating Scale: a cross-sectional study of psychometrics and case finding abilities in general practice. BMC Psychiatry 2005;5:46.

19. Simard S, Savard J. Fear of Cancer Recurrence Inventory: development and initial validation of a multidimensional measure of fear of cancer recurrence. Support Care Cancer 2009;17:241-51.

20. Shin J, Goo A, Ko H, Kim JH, Lim SU, Lee HK, et al. Validation study for the Korean version of Fear of Cancer Recurrence Inventory. J Korean Med Sci 2017;32:1792-9.

21. Pouchieu C, Fassier P, Druesne-Pecollo N, Zelek L, Bachmann P, Touillaud M, et al. Dietary supplement use among cancer survivors of the NutriNet-Sante cohort study. Br J Nutr 2015;113:1319-29.

22. Ambrosone CB, Zirpoli GR, Hutson AD, McCann WE, McCann SE, 
Barlow WE, et al. Dietary supplement use during chemotherapy and survival outcomes of patients with breast cancer enrolled in a cooperative group clinical trial (SWOG S0221). J Clin Oncol 2020;38:804-14.

23. Aoshima E, Mibu M, Kubota M, Hashida T. Use of supplements by Japanese cancer patients receiving outpatient cancer chemotherapy. J Altern Complement Med 2018;24:1003-6.

24. Hauer-Jensen M, Denham JW, Andreyev HJ. Radiation enteropathy: pathogenesis, treatment and prevention. Nat Rev Gastroenterol Hepa- tol 2014;11:470-9.

25. Sohl SJ, Weaver KE, Birdee G, Kent EE, Danhauer SC, Hamilton AS. Characteristics associated with the use of complementary health approaches among long-term cancer survivors. Support Care Cancer 2014;22:927-36.

26. Gansler T, Strollo S, Fallon E, Leach C. Use of complementary/integrative methods: cancer survivors' misconceptions about recurrence prevention. J Cancer Surviv 2019;13:418-28. 